\title{
THE MOST METAL-RICH DWARF STARS IN THE GALACTIC DISK
}

\author{
SOFIA FELTZING \\ Royal Greenwich Observatory $\mathcal{B}$ Insitute of Astronomy, \\ Cambridge, U.K.
}

The metal-rich dwarf stars in the solar neighbourhood have historically not attracted as much attention as the metal-poor (halo) stars which tell us about the early phases of the chemical evolution of our galaxy. The properties of metal-rich stars are highly important when we try to interpret integrated spectra from metal-rich stellar populations, such as the Bulge and elliptical galaxies. A small group of so called super metal rich stars (SMR) have played a significant role in shaping the conceptions of such populations. Famous examples are the dwarf HR1614 and the giant $\mu$ Leonis. In the review by Taylor (1996) SMR stars are discussed in great detail, in particular the reality of extremly high $[\mathrm{Fe} / \mathrm{H}]$. Taylor (1996) find that no giant stars fulfil the criteria fulr SMRness that he sets and only a handful of dwarf stars do, and most of them are possible candidates rather than firm detections.

A few recent studies have studied known SMR candidates and stars with high [M/H] (as derived from photometry), Feltzing \& Gustafsson (1997), Castro et al. (1997), Gonzalez (1997), McWilliam \& Rich (1994), Castro et al. (1996). A first conclusion is that there are indeed quite a few stars with $[\mathrm{Fe} / \mathrm{H}]>0.2 \mathrm{dex}$ (the definition of SMR, see Taylor 1996). We will here review these studies in some detail.

The combination of abundance ratios with kinematical data can detail the picture even further. For example we can study stars on highly eccentric orbits which trace the evolution in the galactic disk closer to the galctic centre. Not much is known about these stars but we did have some very intriguing observations; Barbuy \& Grenon (1990) (hereafter BG90) found that dwarf stars on very eccentric orbits contained much more oxygen than what was expected from standard models of galactic chemical evolution of the disk, Edvardsson et al.(1993) (hereafter E93) found large spreads and "upturns" for certain elements, $\mathrm{Na}, \mathrm{Si}, \mathrm{Ti}, \mathrm{Al}$, for stars with $0.0 \mathrm{dex}<[\mathrm{Fe} / \mathrm{H}]<0.2 \mathrm{dex}$.

\section{Feltzing \& Gustafsson (1997)}

To further explore the questions sketched above we studied $47 \mathrm{G}$ and $\mathrm{K}$ dwarf stars with photometric metallicities $>0.1$ dex. Magnitudes and metallicities were combined with accurate velocity data (UVW) in the selection of stellar samples. One sample trace the chemical evolution in the disk interior to the Sun, $V_{\mathrm{LSR}}<-50 \mathrm{~km} / \mathrm{s}$ and $/$ or $Q_{\mathrm{LSR}}>60 \mathrm{~km} / \mathrm{s}\left(\right.$ where $\left.Q_{\mathrm{LSR}}=\sqrt{U_{\mathrm{LSR}}^{2}+V_{\mathrm{LSR}}^{2}+W_{\mathrm{LSR}}^{2}}\right)$. $V_{\mathrm{LSR}}<-50 \mathrm{~km} / \mathrm{s}$ singles out stars with mean perigalactica less than $7 \mathrm{kpc}$. Another set of stars represent the solar orbit, $Q_{\mathrm{LSR}}<30 \mathrm{~km} / \mathrm{s}$. To explore the oxygen abundances in metal-rich disk dwarfs on highly eccentric orbits we added 3 stars from the sample from BG90. High resolution, $\mathrm{R} \sim 80000$, and high $\mathrm{S} / \mathrm{N}, \sim 200$, spectra of the stars were obtained with the $2 \mathrm{~d}$-coude echelle spectrograph at the $2.7 \mathrm{~m} \mathrm{McDonald} \mathrm{telescope.} \mathrm{For} \mathrm{full} \mathrm{details} \mathrm{of} \mathrm{selection} \mathrm{and} \mathrm{abundance} \mathrm{analysis}$ (standard LTE) see FG97. The abundance ratios are presented in Fig. 1.

We find that the relative abundances of $\mathrm{O}, \mathrm{Na}, \mathrm{Si}$, and $\mathrm{Ti}$ relative to Fe do not vary with the star's present mean distance to the galactic centre (see e.g. Fig. ??). These results imply that there is no significant difference in the chemical evolution of the different stellar populations for stars with $[\mathrm{Fe} / \mathrm{H}]>0$ dex. We find that $[\mathrm{O} / \mathrm{Fe}]$ continues to decline with increasing $[\mathrm{Fe} / \mathrm{H}]$, Fig. 2a, while $[\mathrm{Si} / \mathrm{Fe}]$ and $[\mathrm{Ca} / \mathrm{Fe}]$, however, seem to stay constant. A real ("cosmic") scatter in [Ti/Fe] at a given $[\mathrm{Fe} / \mathrm{H}]$ is suggested as well as a decreasing abundance of the s-elements relative to Fe for the most metal-rich dwarfs. The "upturn" in $[\mathrm{Na} / \mathrm{Fe}]$ vs $[\mathrm{Fe} / \mathrm{H}]$ found by $\mathrm{E} 93$ is confirmed and reinforced, but its origin is still uncertain (metallicity dependent supernova yields may play an important role). We find no such trends for $\mathrm{Al}$ or $\mathrm{Mg}$. 

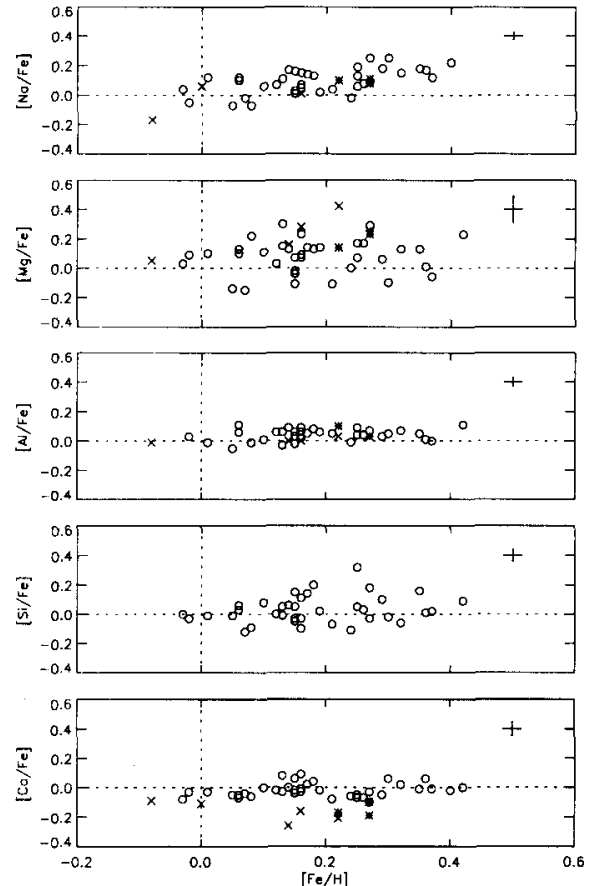
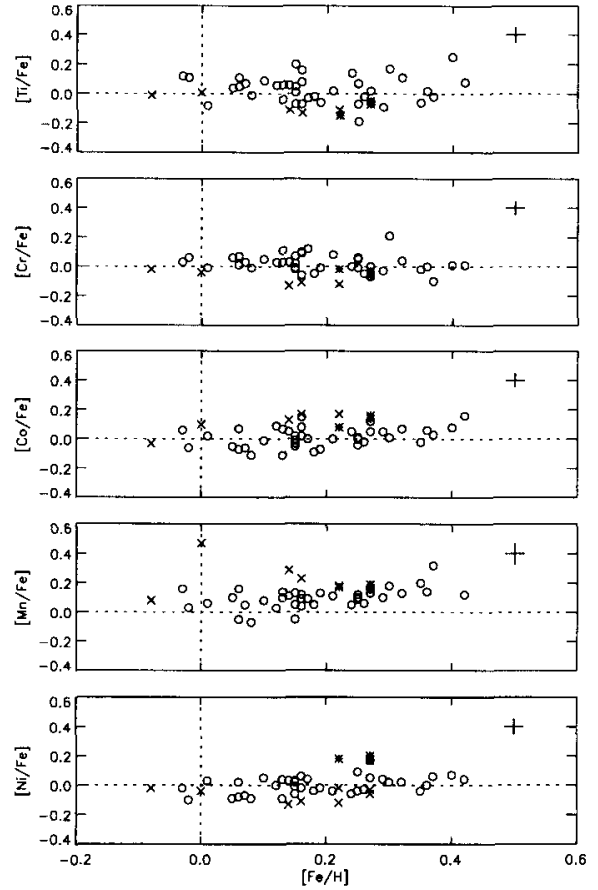

Figure 1. Abundances from Feltzing \& Gustafsson (1997) relative to Fe. $\times$ symbols denote the five $\mathrm{K}$ dwarf stars while * symbols denote the stars in common with Barbuy \& Grenon (1990).

An interesting question is now what difference in $[\mathrm{Na} / \mathrm{Fe}]$ one would have reason to expect for stars formed at different $R_{\mathrm{m}}$. Using the data of Edvardsson et al. (1993) we estimate that the minirnum value of $d[\mathrm{Na} / \mathrm{Fe}] / \mathrm{dR}_{\mathrm{m}}$ is about $0.05 \mathrm{dex} / \mathrm{kpc}$. The results obtained by $\mathrm{FG} 97$ supports this and indicate that the difference in $[\mathrm{Na} / \mathrm{Fe}]$ is, in fact, at the most 0.05 dex.

In this connection, one should also note that orbital diffusion may well mask the possible differences between stars formed at $6 \mathrm{kpc}$ and $8 \mathrm{kpc}$. E.g., stars of solar age in an orbit with $R_{\mathrm{m}}=8.5 \mathrm{kpc}$ may have migrated from an orbit with $R_{\mathrm{m}}=6-7 \mathrm{kpc}$ (cf. Wielen et al. 1996). The mixture of stars with different original orbits, in combination with a radial galactic gradient of $[\mathrm{Fe} / \mathrm{H}]$, was proposed by Wielen et al. (1996) to explain the unexpectedly high scatter in $[\mathrm{Fe} / \mathrm{H}]$ of 0.2 dex for solar type stars, with similar age and similar present $R_{\mathrm{m}}$, found by Edvardsson et al. (1993). From the work of Wielen et al. (1996) we estimate that two samples of stars with $\left\langle R_{\mathrm{m}}\right\rangle=8.5$ and $6.5 \mathrm{kpc}$, respectively, would then be mixed by orbital diffusion so much that the population effects in abundances only would show up to about half the expected size as compared with the situation if orbital diffusion is not present. Although the reason for the great inhomogeneities in the gravitational potential, needed to account for the orbital diffusion of this magnitude, is not known, we conclude that the effects looked for by dividing the total sample of stars according to the velocity criteria used here, might be diminished considerably by this phenomenon.

\section{Other studies}

Barbuy \& Grenon (1990) studied oxygen abundances for 11 dwarf stars on highly eccentric orbits. They find the stars to be enriched in oxygen as compared to expectations from models of galactic chemical evolution for stars of this metallicity, Fig. $2 a$.

Edvardsson et al. (1993): in their total sample of 189 dwarf stars, 53 have $[\mathrm{Fe} / \mathrm{H}]>0.0 \mathrm{dex}$ (out of which 4 are above $0.2 \mathrm{dex}$ ). They found interesting features, for these stars, such as the upturns in $\mathrm{Na}, \mathrm{Mg}$ and $\mathrm{Al}$. They also found large spreads for certain elements, $\mathrm{Si}, \mathrm{Mg}, \mathrm{Ti}$. The 

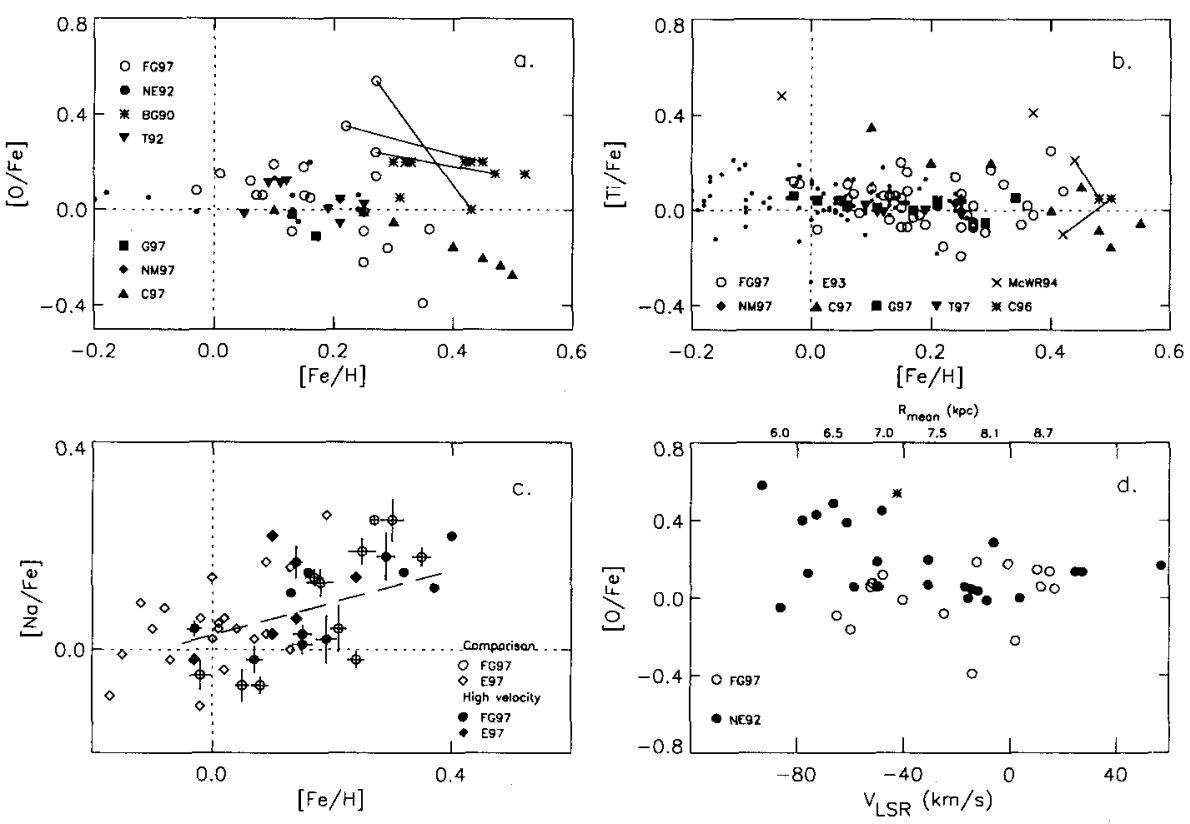

Figure 2. a. Oxygen abundances from several studies. Results for the three stars studied by both FG97 and BG90 are connected with full lines. b. Titanium abundances from several studies. Giant stars studied by both McWR94 and $\mathrm{C96}$ are connected with full lines. $\mu$ Leo is the star with highest $[\mathrm{Fe} / \mathrm{H}]$ in the study by C96. c. The sodium data from FG97 devided into subsamples and compared with samples from E93 selected in the same way as for FG97. The dashed line indicates a least-square fit to all the stars in FG97. Errorbars are showed for the stars from FG97. d. Oxygen abundances from FG97 and NE92 as a function of $V_{L S R}$. $V_{L S R}$ can be translated to mean distance to galactic centre. Distances are indicated on the top of the diagram. Acronyms used in the figures; BG90 = Barbuy \& Grenon (1990), C96 = Castro et al. (1996), C97 = Castro et al. (1997), E93 = Edvardsson et al. (1993), FG97 = Feltzing \& Gustafsson (1997), G97 = Gonzalez (1997abc), McWR94 = McWilliam \& Rich (1994), NE = Nissen \& Edvardsson (1992), NM97 = Neuforge \& Magain (1997), T97 = Tomkin et al. (1997).

question was raised weather or not this was a cosmic spread. E93 found a group of stars enriched in $\mathrm{Fe}$ and in $\mathrm{Na}, \mathrm{Mg}$ and $\mathrm{Al}$, dubbed the $\mathrm{NaMgAl}$ stars. Tomkin et al. (1997) re-investigated, with new spectra, nine of the stars studied in E93, six of which had been identified as NaMgAl stars. They find no evidence for these stars to be enriched in $\mathrm{Na}, \mathrm{Mg}$ or Al. This is, for $\mathrm{Na}$, contradictory to the findings of FG97. The main conclusion, however, is that there does not exist a group of stars enriched in $\mathrm{Na}, \mathrm{Mg}$ and $\mathrm{Al}$.

Further results on metal-rich dwarfs have been published by Gonzalez (1997abc), NeuforgeVerheecke \& Magain (1997) ( $\alpha$ Centauri System) and Castro et al. (1997). The results from these studies nicely confirm the findings by FG97, Fig. 2a and b.

Gonzalez has explored the link between high metallicity in planetary system candidates (e.g. $\rho^{1} 55 \mathrm{Cnc}, 51 \mathrm{Peg}$ ). Tentative results from his investigations point to a correlation between metalrichness and the presence of planets.

Castro et al. (1997) investigated 9 stars selected in the same way as for BG90, i.e. very metalrich stars on highly excentric orbits. From Geneva photometry their metallicities are between +0.3 and $+0.6 \mathrm{dex}$; this is confirmed by the abundance analysis. A mixture of oscillator strengths are used. In general their results confirm the findings by FG97. One would expect these stars to show a similar abundance pattern for $\mathrm{O}$ as the BG90 stars. This is not the case, see Fig. 2a. While the BG90 stars clearly are overabundant in oxygen these additional 9 stars seem to be underabundant and follow the same trend as FG97. $[\mathrm{Na} / \mathrm{Fe}]$ is $\sim 0.5$ dex higher than for the stars studied by E93 and $\mathrm{FG} 97$ in the same $[\mathrm{Fe} / \mathrm{H}]$ range. The reason for this is unclear. It is also not possible to directly address the question of abundances as a function of velocity, since velocity data for these stars have not yet been published. 
Here we will only discuss two studies of giant stars. McWilliam \& Rich (1994) derived abundances for 12 giant bulge stars. This work clearly showed that high dispersion is necessary for derivation of reliable abundances in giants. The McWilliam \& Rich (1994) study suggests that the metallicity of the stars in the Bulge is, in the mean, slightly less than solar and with a large spread and that $\mathrm{Si}$ and $\mathrm{Ti}$ may be overabundant as compared to the local disk stars. On the other hand the Castro et al. (1996) study (at still higher resolution) of two giants, one from McWilliam \& Rich (1994) and $\mu$ Leo, show the stars to be very similar to disk dwarfs of the same [Fe/H], Fig. $2 b$.

\section{Concluding remarks}

To conclude we are most probably seeing, in the most metal-rich disk dwarfs, the natural end of "normal" galactic chemical evolution in the disk. The abundance trends of [Element/Fe] vs $[\mathrm{Fe} / \mathrm{H}$ ] up to $[\mathrm{Fe} / \mathrm{H}]=0.2$ dex are now well established for a large number of elements $(\mathrm{O}, \mathrm{Na}, \mathrm{Mg}, \mathrm{Al}, \mathrm{Ca}$, $\mathrm{Ni}$ etc). These trends fit nicely into the overall picture of chemical evolution in the galactic disk as it is currently understood, apart from, possibly, $\mathrm{O}, \mathrm{Na}, \mathrm{Si}$ and $\mathrm{Ti}$.

The full power of combining abundance data and kinematical data in studies of stellar populations has not yet been fully exploited, nor have the particular problems that may hamper such investigations, e.g. migration and its origin, been put on a sure footing. This needs to be done before data for stars on highly eccentric orbits can be reliably interpreted.

We cannot, so far, infer much about the bulge population(s) from locally studied stars. This may not even be possible, but nevertheless studies of stars on highly eccentric orbits are of great interest since they may tell us about the evolution of the inner disk. This in combination with future direct studies of abundances in the bulge stars will help to discriminate between different formation scenarios for bulges, i.e. are they separate populations related to the disks (suggested by colours of disks and bulges Balcells and Peletier 1996) or halos (suggested by kinematics e.g. Ibata and Gilmore 1995). For the time being we can only conclude that the bulge giants (McWilliam \& Rich 1994) do not look any different in terms of abundance ratios, at a given $[\mathrm{Fe} / \mathrm{H}]$, than do the local dwarfs (taking the errors into account) and that the abundance trends for stars from the inner disk are highly uncertain and have to be confirmed before one can make any firm conclusions on the relation between disks and bulges in spiral galaxies.

Spectra of such metal-rich stars as discussed here are very crowded and there is a clear need for further studies at high resolution and high signal to noise so that continua can be reliably defined and blends excluded. This is now possible for large samples of local dwarf stars, as demonstrated by E93 and FG97. For giant stars in the Bulge the new spectrographs on $8 \mathrm{~m}$ telescopes (e.g. UVES on VLT) will make it possible to observe larger samples of stars in reasonable integration times. In particular we should study the metal-rich dwarf stars on highly eccentric orbits and the Bulge giants to gain clues to the formation scenario of the Bulge and bulges in other galaxies. We also need to build up statistics for local dwarfs with $[\mathrm{Fe} / \mathrm{H}]$ between 0.2 and 0.4 dex and to confirm or disconfirm the very highest iron abundances derived.

\section{References}

Balcells \& Peletier, 1996, $A J, 111,2238$

Barbuy B., Grenon M., 1990, in Bulges of Galaxies, ESO/CTIO workshop, ed. Jarvis, B.J., Terndrup, D.M., 83

Castro S., Rich R.R., Grenon M., et al., 1997, $A J, 114,376$

Castro S. Rich R.R., McWilliam A., et al. 1996, $A J, 111,2439$

Edvardsson B., Andersen J., Gustafsson B., et al., 1993, $A \& A, 275,101$

Feltzing S., Gustafsson B., 1997, $A \mathcal{E} A S$, accepted

Gonzalez G., 1997a, PASP, in press

Gonzalez G., 1997b, preprint, to appear in proceedings of "Brown Dwarfs and Extrasolar Planets Workshop"

Gonzalez G., 1997c, MNRAS, 285, 403

Ibata R., Gilmore G., 1995, MNRAS, 275, 605

Mcwilliam A., Rich M., 1994, ApJS, 91, 749

Neuforge-Verheecke C., Magain, P., 1997, $A \& A$, accepted

Taylor B.J., 1996, ApJS, 102, 105

Tomkin J., Woolf V.M., Lambert D.L., Lemke M., 1995, AJ, 109, 2204

Wielen R., Fuchs B., Dettbarn C., 1996, A $B A, 314,438$ 